IMAGES IN NEONATAL MEDICINE

\title{
Neonatal gastrointestinal perforation
}

$\mathrm{N}$

enatal gastrointestinal perforation is a common condition carrying a mortality of $17-60 \%{ }^{1}$ Clinical suspicion is supported by radiological signs, which may be subtle and must be sought specifically. We review these signs in the light of several recent instances of delayed recognition of intestinal perforations, one of which is described here.

A 24 day old preterm infant was referred to our centre for ligation of a patent ductus arteriosus after failed indomethacin treatment. The infant developed abdominal distension and bilious aspirates the following day. The abdominal radiograph at this stage was deemed "unremarkable".

In view of clinical deterioration and persistent abdominal distension, a contrast study was performed. A small leakage of contrast into the right iliac fossa indicated a perforation. Review of previous radiographs from the referring centre (which had been temporarily mislaid) dislosed a classic football sign ( fig 1): the central lucency had not been noted as the film had been intended to check the position of a percutaneous line. Laparotomy disclosed an isolated perforation of the terminal ileum and longstanding peritoneal contamination.

Neonatal gastrointestinal perforation most commonly occurs as a complication of necrotising enterocolitis ( $42 \%$ of cases), where it is associated with a high mortality of $62 \% .^{2}{ }^{3}$ Spontaneous or idiopathic perforation, usually involving the terminal ileum, is the next most common presentation in $22 \%,{ }^{2}$ and is associated with a lower mortality of $14 \%$.

Evidence of free air on abdominal radiograph often tips the balance between conservative management and operative treatment. A review of 105 cases of perforated necrotising enterocolitis found that abdominal radiographs show evidence of free intraperitoneal gas in only $63 \%$ of cases, with $21 \%$ showing ascites but not free air, and $16 \%$ showing neither feature. ${ }^{4} \mathrm{~A}$ confounding factor is that a pneumoperitoneum could also be due to air tracking from the chest in ventilated patients. Radiographs may need to be repeated frequently in order to pick up tell tale signs as early as possible.

Several signs are pathognomonic of pneumoperitoneum, regardless of the age group. ${ }^{5}$ A common sign is a collection of gas in the right upper quadrant adjacent to the liver, lying mainly in the subhepatic space and the hepatorenal fossa, which is visible as an oval or triangular gas shadow not obviously in continuity with the rest of the bowel ${ }^{6}$ (fig 2). Clear visualisation of the outer as well as the inner wall of a bowel loop-Rigler's sign-is a valuable indication of a pneumoperitoneum ${ }^{6}$ (fig 2). Small triangular collections of gas between loops of bowel may sometimes be identified. ${ }^{6}$

Reflections of the peritoneum normally present on the inner surface of the anterior abdominal wall are not usually identified, but may be visualised by free gas when it lies on either side. Thus the falciform ligament in particular, and occasionally the umbilical ligaments and urachus, may sometimes be identified in the presence of free air (figs 2 and 3). The falciform ligament appears as a white streak, and when surrounded by the oval lucency of a pneumoperitoneum, supposedly resembles the seam of an American football. Hence the "football sign", visible on supine view (fig 1). Relatively large amounts of gas may accumulate and show up beneath the diaphragm (cupola sign), or on the upper part of the abdomen in a decubitus or "shootthrough" lateral film. A gasless abdomen may also represent perforation.

Ultrasonography may be help to assess the presence and the character of ascites, and is particularly helpful in the context of a gasless abdomen. The presence of particulate matter most likely indicates perforation.

Early diagnosis of intestinal perforation is imperative to allow prompt surgical intervention. In the absence of expert radiological opinion, a low threshold for performing decubitus films with the right side up is advised, as free gas is most easily perceived separate from other bowel, lateral to the liver. A systematic review of all radiographs is advisable so that signs are not missed, especially when films are taken to assess positions of tubes and lines. An awareness of the clinical presentation and main radiological signs will enable the diagnosis to be made more promptly even in centres without paediatric surgical expertise.

M K Farrugia, A S Morgan, K McHugh, E M Kiely Great Ormond St Hospital for Sick Children, London, UK; mkfarrugia@hotmail.com

\section{References}

1 Tan CE, Kiely EM, Agrawal M, et al. Neonatal gastrointestinal perforation. J Pediatr Surg 1989;24:888-92.

2 Grosfeld JL, Molinari F, Chaet M, et al. Gastrointestinal perforation and peritonitis in infants and children. An experience with 179 cases over 10 years. Surgery 1996;120:650-5.

3 Zamir O, Shapira SC, Udassin R, et al. Gastrointestinal perforations in the neonatal period. Am J Perinatol 1988;5:131-3.

4 Frey EE, Smith W, Franken EA, et al. Analysis of bowel perforation in necrotizing enterocolitis. Pediatr Radiol 1987;17:380-2.

5 Miller RE. Perforated viscus in infants: a new roentgen sign. Radiology 1960;74:65-7.

6 Rigler LG. Spontaneous pneumoperitoneum: a roentgenological sign found in the supine position. Radiology 1942;20:604-7.

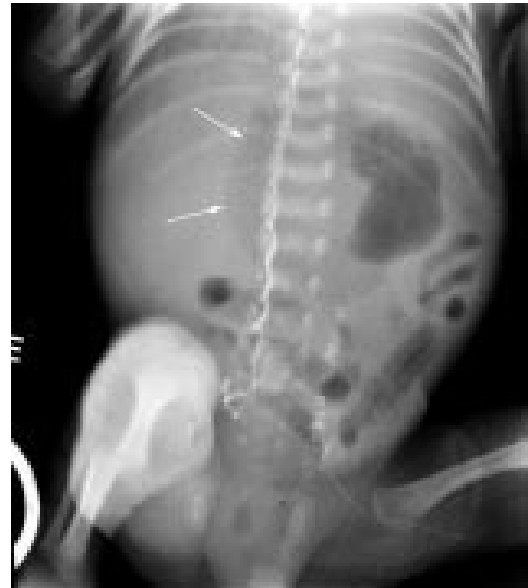

Figure 1 Intraperitoneal air visible as a central oval lucency: the football sign.

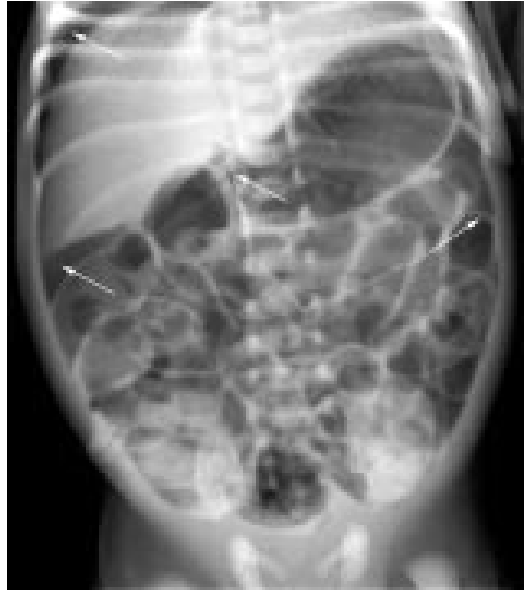

Figure 2 Abdominal radiograph showing free subdiaphragmatic and subhepatic air falciform ligament outlined by intraperitoneal air; Rigler's Sign in upper left quadrant (arrowed).

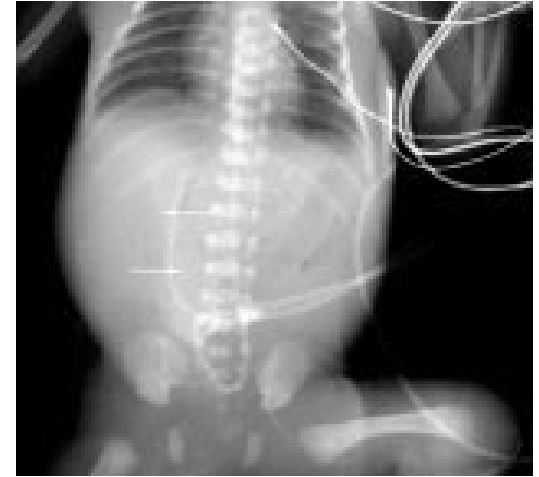

Figure 3 Free air outlining falciform ligament (arrowed). 